\title{
Approximate method for solving strongly fractional nonlinear problems using fuzzy transform
}

https://doi.org/10.1515/nleng-2018-0123

Received August 19, 2018; revised November 16, 2018; accepted January 28, 2019.

\begin{abstract}
In this research work, we have shown that it is possible to use fuzzy transform method (FTM) for approximate solution of strongly fractional nonlinear problems. In numerical methods, in order to approximate a function on a particular interval, only a restricted number of points are employed. However, what makes the $F$-transform preferable to other methods is that it makes use of all points in this interval. The comparison of the time used in minutes is given for two derivatives Caputo derivative and CaputoFabrizio derivative.
\end{abstract}

Keywords: Fuzzy transform; Riccati differential equations; Bratu differential equations; Caputo derivative; CaputoFabrizio derivative

\section{Introduction}

Fractional arithmetic and fractional differential equations appeared in many sciences, including medicine [1], economics [2], dynamical problems [3, 4], chemistry [5], chaotic systems [6], mathematical physics [7-11], traffic model [12], entropy [13] and fluid flow [14] and so on. Scholars and researchers are invited to check books that have been written to take advantage of fractional arithmetic $[15,16]$.

Many researchers have used numerical methods for the purpose of solving the fractional Riccati differential equations (FRDEs) and the fractional Bratu differential equations (FBDEs).

\footnotetext{
Mohamad Adabitabar Firozja, Department of Mathematics, Qaemshahr Branch, Islamic Azad University, Qaemshahr, Iran, Email: m.adabitabar@qaemiau.ac.ir

*Corresponding Author: Bahram Agheli, Department of Mathematics, Qaemshahr Branch, Islamic Azad University, Qaemshahr, Iran, E-mail: b.agheli@qaemiau.ac.ir
}

In this research work, we have for the first time shown that it is possible to use F-transform method (FTM) to tackle with FRDEs and FBDEs of the following forms.

(1) Fractional Riccati differential equations (FRDEs)

$$
\begin{aligned}
& D^{\alpha} u(t)-\sum_{i=0}^{2} p_{i}(t) u^{i}(t)=0, \quad 0<\alpha \leq 1, \\
& 0<t \leq T, \quad u(0)=u_{0},
\end{aligned}
$$

where $t \in \mathbb{R}, p_{i}(t), i=0,1,2$ are constant functions.

(2) Fractional Bratu differential equations (FBDEs)

$$
\begin{aligned}
& D^{\alpha} u(t)-\lambda \exp (u(t))=0, \quad 1<\alpha \leq 2, \\
& 0<t \leq T, \quad u(0)=u_{0}, \quad u_{t}(0)=u_{0}^{\prime},
\end{aligned}
$$

where $\lambda>0$ and $t \in \mathbb{R}, p_{i}(t), i=0,1,2$ are constant functions.

The operator $D^{\alpha}$ denotes the Caputo's derivative [16] of or$\operatorname{der} \alpha$

$$
\begin{aligned}
& D^{\alpha} u(t)=\frac{1}{\Gamma(n-\alpha)} \int_{0}^{t}(t-s)^{\alpha-1} u^{(n)}(s) d s, \\
& t>a, \quad n-1<\alpha \leq n, \quad n \in \mathbb{N}
\end{aligned}
$$

or Caputo-Fabrizio’s derivative [17] of order $\alpha$

$$
\begin{aligned}
& \mathfrak{D}_{t}^{n+\alpha} u(t)=\frac{T(\alpha)}{1-\alpha} \int_{a}^{t} \exp \left(\frac{(s-t) \alpha}{1-\alpha}\right) u^{(n+1)}(s) d s, \\
& t>a, n<\alpha \leq n+1, \quad n \in \mathbb{Z}^{+},
\end{aligned}
$$

in which, $T(\alpha)$ is called, the normalization function featuring $T(0)=T(1)=1$.

Historically, a special case of this differential equation by James Bernoulli (1654-1705) and then by Count Jacopo Francesco Riccati (1676-1754) was introduced and evaluated. On the importance and motivation for this differential equation, it should be noted that it has a key role in many of the physical phenomena and other sciences. Such 
applications can include control systems, robust stabilization, network synthesis, diffusion problems, optimal filtering, controls, stochastic theory, financial mathematics, optimal control, river flows, robust stabilization, network synthesis and financial mathematics dynamic games, linear systems with Markovian jumps, stochastic control, econometric models and invariant embedding noted that the use of the Riccati differential equation [18-25]. Of other uses, the one dimensional static Schrödinger equation [26] and the travelling wave solutions of a nonlinear partial differential equation [27] are noteworthy with the Riccati differential equation featuring fractional derivatives.

On the importance and motivation for Bratu differential equation, it should be noted that it has a key role in many of the physical phenomena, chemical models and other sciences. Such applications can include model of thermal reaction process, the fuel ignition model of the thermal combustion theory, the Chandrasekhar model of the expansion of the universe, radiative heat transfer nanotechnology and chemical reaction theory $[28,29,32,33]$

The FTM has recently been utilized by authors in [3436 ] to find approximate solution of the first order fuzzy differential equations and two-point boundary value problems. Along the same line of research, Chen and his associates in [37] have established an algorithm to gain the numerical solutions of second order primary amount problems.

It must be pointed out here that researchers have utilized disparate schemes to solve FRDEs during the last two decades. We can refer to familiar methods, including differential transform method [38], Adomian's decomposition method [39], homotopic perturbation method [39], variational iteration method [40], homotopic analysis method [41] and etc [42-44]. For numerical solution of FBDEs we can point to homotopic perturbation method [46], optimal homotopy asymptotic method [47] and variational iteration technique [48] and etc. Scholars and researchers are invited to study other numerical solutions in [49-54]

\section{Discretization of the fractional derivative}

Assume that $u(t)$ is the solution to equations (1.1) and (1.2). To calculate the approximation of $u(t)$, we use the discretization of the Caputo derivative and Caputo-Fabrizio derivative with the assumption $\tau=t_{j+1}-t_{j}$ and $t_{j}=a+j \tau$, $j=0,1,2, \cdots$.

\subsection{Discretization of the Caputo derivative}

Utilizing the approximation for the Caputo derivative [56] of Eq. (1.3) we have:

$D^{\alpha} u\left(t_{k+1}\right) \approx$

$\frac{1}{\tau^{\alpha} \Gamma(2-\alpha)} \sum_{j=0}^{k}\left(u\left(t_{j+1}\right)-u\left(t_{j}\right)\right)\left((k-j+1)^{1-\alpha}-(k-j)^{1-\alpha}\right)$,

in which $0<\alpha \leq 1, u\left(t_{0}\right)$ is known and

$$
\begin{aligned}
& D^{\alpha} u\left(t_{k+1}\right) \approx \frac{1}{\tau^{\alpha} \Gamma(3-\alpha)} \sum_{j=0}^{k}\left(u\left(t_{j+1}\right)-2 u\left(t_{j}\right)+u\left(t_{j-1}\right)\right) \times \\
& \left((k-j+1)^{2-\alpha}-(k-j)^{2-\alpha}\right)
\end{aligned}
$$

in which $1<\alpha \leq 2, u\left(t_{0}\right)$ and $u^{\prime}\left(t_{0}\right)$ are known and and $u\left(t_{-1}\right)=u\left(t_{0}\right)-\tau u^{\prime}\left(t_{0}\right)$.

\subsection{Discretization of the Caputo-Fabrizio derivative}

Utilizing the approximation for the Caputo-Fabrizio derivative [57] of Eq. (1.4) we have:

$$
\begin{aligned}
& D^{\alpha} u\left(t_{k+1}\right) \approx \frac{1}{\alpha \tau} \sum_{j=0}^{k}(u(j+1)-u(j)) \times \\
& \quad\left(\exp \left(-\frac{(\alpha \tau)(k-j)}{1-\alpha}\right)-\exp \left(-\frac{(\alpha \tau)(k-j+1)}{1-\alpha}\right)\right),
\end{aligned}
$$

in which $0<\alpha \leq 1, u\left(t_{0}\right)$ is known and

$$
\begin{gathered}
D^{\alpha} u\left(t_{k+1}\right) \approx \frac{1}{\alpha \tau^{2}} \sum_{j=0}^{k}(u(j-1)+u(j+1)-2 u(j)) \times \\
\left(\exp \left(-\frac{\alpha \tau(k-j)}{1-\alpha}\right)-\exp \left(-\frac{\alpha \tau(k-j+1)}{1-\alpha}\right)\right),
\end{gathered}
$$

in which $1<\alpha \leq 2, u\left(t_{0}\right)$ and $u^{\prime}\left(t_{0}\right)$ are known and $u\left(t_{-1}\right)=$ $u\left(t_{0}\right)-\tau u^{\prime}\left(t_{0}\right)$.

\section{Fuzzy partition and fuzzy transform}

In this section, only the main definitions of $F$-transform to be utilized in the subsequent sections of numerical implementations will be outlined. 
Definition 3.1. [55] Presuming that for $n \geq 2, a=t_{1}<t_{2}<$ $\cdots<t_{n-1}<t_{n}=b$ be specified nodes, we express that fuzzy sets $B_{1}, \cdots, B_{n}$ defined on $[a, b]$ with their membership functions $B_{1}(t), \cdots, B_{n}(t)$, form a fuzzy partition of $[a, b]$ if they meet the following properties:

(1) $B_{k}$ of $[a, b]$ to $[0,1]$ is continuous, $\sum_{k=1}^{n} B_{k}(t)=1$ for all $t \in[a, b]$ and $B_{k}\left(t_{k}\right)=1, k=1,2, \cdots, n$.

(2) $B_{k}(t)=0$ if $t \notin\left(t_{k-1}, t_{k+1}\right)$, with $t_{0}=a$ and $t_{n+1}=b$,

(3) On subinterval $\left[t_{k-1}, t_{k+1}\right]$, for $k=2, \cdots, n-1, B_{k}(t)$, certainly is an increasing function on $\left[t_{k-1}, t_{k}\right]$ and decreasing function on $\left[t_{k}, t_{k+1}\right]$.

The membership functions $B_{1}, B_{2}, \cdots, B_{n}$ are named basic functions (BFs).

The next formulas give the standard display of such triangular membership functions:

$B_{1}(t)= \begin{cases}1-\frac{t-t_{1}}{h_{1}}, & t_{1} \leq t \leq t_{2} \\ 0, & \text { otherwise }\end{cases}$
$B_{k}(t)= \begin{cases}\frac{t-t_{k-1}}{h_{k-1}}, & t_{k-1} \leq t \leq t_{k} \\ 1-\frac{t-t_{k}}{h_{k}}, & t_{k} \leq t \leq t_{k+1}, k=2,3, \cdots, n-1, \\ 0, & \text { otherwise, }\end{cases}$

$B_{n}(t)= \begin{cases}\frac{t-t_{n-1}}{h_{n-1}}, & t_{n-1} \leq t \leq t_{n}, \\ 0, & \text { otherwise. }\end{cases}$

The formulas that follow for $k=2, \cdots, n-1$ give the standard display of such sinusoidal membership functions:

$B_{1}(t)=\left\{\begin{array}{lc}0.5\left(1+\cos \frac{\pi}{h}\left(t-t_{1}\right)\right), & t_{1} \leq t \leq t_{2} \\ 0, & \text { otherwise, }\end{array}\right.$
$B_{k}(t)= \begin{cases}0.5\left(1+\cos \frac{\pi}{h}\left(t-t_{k}\right)\right), & t_{k-1} \leq t \leq t_{k+1}, \\ k=2,3, \cdots, n-1, & \text { otherwise, }\end{cases}$

$B_{n}(t)=\left\{\begin{array}{lr}0.5\left(1+\cos \frac{\pi}{h}\left(t-t_{n}\right)\right), & t_{n-1} \leq t \leq t_{n} \\ 0, & \text { otherwise, }\end{array}\right.$

in which $h_{k}=t_{k+1}-t_{k}$ for $k=1, \cdots, n-1$. It can be stated that fuzzy partition of $[a, b]$, is uniform if $t_{k+1}-$ $t_{k}=h=\frac{b-a}{n-1}$ and two additional properties coincide:

(4) $B_{k}\left(t_{k}-t\right)=B_{k}\left(t_{k}+t\right)$, for all $t \in[0, h]$, for $k=2, \cdots, n-$ 1 ,

(5) $B_{k}(t)=B_{k-1}(t-h)$ and $B_{k+1}(t)=B_{k}(t-h)$, for $k=$ $2, \cdots, n-1$, and $t \in\left[t_{k}, t_{k+1}\right]$.

Definition 3.2. [55] Let $f$ be any function belonging to $C([a, b])$ and $B_{1}, B_{2}, \cdots, B_{n}$, be the $B F s$ which buildup a fuzzy partition of $[a, b]$. We define the $n$-tuple
$\left[F_{1}, F_{2}, \cdots, F_{n}\right]$ of real numbers given by

$$
F_{k}=\frac{\int_{a}^{b} f(t) B_{k}(t) d t}{\int_{a}^{b} B_{k}(t) d t}, \quad k=1,2, \cdots, n,
$$

as the $F$-transform of $f$ in relation to $B_{1}, B_{2}, \cdots, B_{n}$.

Definition 3.3. [55] Let $\left[F_{1}, F_{2}, \cdots, F_{n}\right]$ be the $F$-transform of function $f$ relative to $B F s, B_{1}, B_{2}, \cdots, B_{n}$. Then,

$$
f_{n}(t)=\sum_{k=1}^{n} F_{k} B_{k}(t)
$$

is named the inverse $F$-transform (IFT) of function $f$ on $[a, b]$.

Theorem 3.4. [55] Let $f$ be a continuous function on $[a, b]$ and $B_{1}, B_{2}, \cdots, B_{n}$ be the $B F s$ which form a fuzzy partition of $[a, b]$. Then, the kth component of the integral $F$-transform signified over $[f(a), f(b)]$, gives the minimum to the function

$$
\phi(y)=\int_{a}^{b}(f(t)-y)^{2} B_{k}(t) d t .
$$

Lemma 3.5. [55] (Convergence) Let $f$ be a continuous function on $[a, b]$. Thus, for any $\epsilon>0$, there exist $n_{\epsilon}$ and a fuzzy partition $B_{1}, \cdots, B_{n_{\epsilon}}$ of $[a, b]$ such that for all $t \in[a, b]$

$$
\left|f(t)-f_{n_{\epsilon}}(t)\right| \leq \epsilon .
$$

\section{Description of the new approach}

Let $u(t)$ be the continuous solution of (1.1) on [0,T]. Also, $U_{1}, \cdots, U_{n}$ of F-transform $u(t)$, calculated by using BFs $B_{0}, B_{1}, \cdots, B_{n}$ in $[0, T]$ regarding (3.1) with $t_{j+1}-t_{j}=$ $\tau$ which are uniform fuzzy partitions. Now with applying IFT on the function $u(t)$ give the approximation $u_{n}(x)$ as according to:

$$
u_{n}(t)=\sum_{k=0}^{n} U_{k} B_{k}(t), \quad t \in[0, T] .
$$

Hence for approximate solution, we can calculated $U_{k}$ for $k=0,1,2, \cdots, n$, where $U_{k}$, are not $F$-transform of $u$ and must be calculated.

In the next proposition the discretization of the Caputo derivative for $u_{n}(t)$ for Eqs.(2.1),(2.2), (2.3) and (2.4) are presented. 
Proposition 4.1. With substituting $u_{n}(t)$ in Eqs.(2.1),(2.2), (2.3) and (2.4), we will have the next equations, respectively:

$$
\begin{aligned}
& D^{\alpha} u_{n}\left(t_{k+1}\right) \approx \\
& \frac{1}{\tau^{\alpha} \Gamma(2-\alpha)} \sum_{j=0}^{k}\left(U_{j+1}-U_{j}\right)\left((k-j+1)^{1-\alpha}-(k-j)^{1-\alpha}\right), \\
& 0<\alpha \leq 1, \\
& D^{\alpha} u_{n}\left(t_{k+1}\right) \approx \frac{1}{\tau^{\alpha} \Gamma(3-\alpha)} \sum_{j=0}^{k}\left(U_{j+1}-2 U_{j}+U_{j-1}\right) \times \\
& \quad\left((k-j+1)^{2-\alpha}-(k-j)^{2-\alpha}\right), 1<\alpha \leq 2, \\
& D^{\alpha} u_{n}\left(t_{k+1}\right) \approx \frac{1}{\alpha \tau} \sum_{j=0}^{k}\left(U_{j+1}-U_{j}\right) \times \\
& \quad\left(\exp \left(-\frac{(\alpha \tau)(k-j)}{1-\alpha}\right)-\exp \left(-\frac{(\alpha \tau)(k-j+1)}{1-\alpha}\right)\right), \\
& 0<\alpha \leq 1, \\
& D^{\alpha} u_{n}\left(t_{k+1}\right) \approx \frac{1}{\alpha \tau^{2}} \sum_{j=0}^{k}\left(U_{j+1}-2 U_{j}+U_{j-1} \times\right. \\
& \left(\exp \left(-\frac{(\alpha \tau)(k-j)}{1-\alpha}\right)-\exp \left(-\frac{(\alpha \tau)(k-j+1)}{1-\alpha}\right)\right),
\end{aligned}
$$$$
1<\alpha \leq 2 \text {, }
$$

where $u\left(t_{0}\right)$ and $u^{\prime}\left(t_{0}\right)$ are known of initial conditions, $U_{0}=u\left(t_{0}\right)$ and $U_{-1}=u\left(t_{0}\right)-\tau u^{\prime}\left(t_{0}\right)$.

\subsection{Approximate solution of FRDEs}

In order to gain the approximate solution of the problem (1.1), we use of $u_{n}(t)$, hence

$$
D^{\alpha} u_{n}(t)=\sum_{i=0}^{2} p_{i}(t) u_{n}^{i}(t), \quad 0<\alpha \leq 1, \quad 0<t \leq T,
$$

and by putting $t=t_{k+1}$, we have

$$
\begin{aligned}
& D^{\alpha} u_{n}\left(t_{k+1}\right)=\sum_{i=0}^{2} p_{i}\left(t_{k+1}\right) u_{n}^{i}\left(t_{k+1}\right), \\
& 0<\alpha \leq 1, \quad k=0,1, \cdots, n-1 .
\end{aligned}
$$

Case 1. Considering Caputo's derivative: using Eq.(4.2), Eq.(4.7) convert to the following form

$$
\frac{1}{\tau^{\alpha} \Gamma(2-\alpha)} \sum_{j=0}^{k}\left(U_{j+1}-U_{j}\right)\left((k-j+1)^{1-\alpha}-(k-j)^{1-\alpha}\right)
$$

$$
=\sum_{i=0}^{2} p_{i}\left(t_{k+1}\right) U_{k+1}^{i}, k=0,1,2, \cdots, n-1 .
$$

Case 2. Considering Caputo-Fabrizio derivative: using Eq.(4.4), Eq.(4.7) convert to the following form

$$
\begin{aligned}
& \frac{1}{\alpha \tau} \sum_{j=0}^{k}\left(U_{j+1}-U_{j}\right)\left(\exp \left(-\frac{(\alpha \tau)(k-j)}{1-\alpha}\right)\right. \\
& \left.-\exp \left(-\frac{(\alpha \tau)(k-j+1)}{1-\alpha}\right)\right)=\sum_{i=0}^{2} p_{i}\left(t_{k+1}\right) U_{k+1}^{i},
\end{aligned}
$$

for $k=0,1,2, \cdots, n-1$.

Now, using the boundary condition, we can calculate $U_{1}, U_{2}, \cdots, U_{n}$ by recursive equation and then by $I F T$ gain the approximate solution $u(t) \approx u_{n}(t)$ for Eq.(1.1). An algorithm for approximation of FRDEs by this method stated in the next Algorithm.

Algorithm 1. An approximation algorithm for FRDEs

Step 1. Input $p_{0}(t), p_{1}(t), p_{2}(t), U_{0}=u(0), n$ and $T$.

Step 2. Set $\tau \leftarrow \frac{T}{n}$.

Step 3. Locate $t_{k} \leftarrow k \tau, k=0,1,2, \cdots, n$.

Step 4. Choose sinusoidal $B F s B_{k}(t)$ for $k=0,1,2, \cdots, n$.

Step 5. a) With Caputo derivative, set recursive equation

$$
\begin{aligned}
& \frac{1}{\tau^{\alpha} \Gamma(2-\alpha)} \sum_{j=0}^{k}\left(U_{j+1}-U_{j}\right)\left((k-j+1)^{1-\alpha}-(k-j)^{1-\alpha}\right) \\
& =\sum_{i=0}^{2} p_{i}\left(t_{k+1}\right) U_{k+1}^{i}
\end{aligned}
$$

b) With Caputo-Fabrizio derivative, set recursive equation

$$
\begin{aligned}
& \frac{1}{\alpha \tau} \sum_{j=0}^{k}\left(U_{j+1}-U_{j}\right)\left(\exp \left(-\frac{(\alpha \tau)(k-j)}{1-\alpha}\right)\right. \\
& \left.-\exp \left(-\frac{(\alpha \tau)(k-j+1)}{1-\alpha}\right)\right)=\sum_{i=0}^{2} p_{i}\left(t_{k+1}\right) U_{k+1}^{i} .
\end{aligned}
$$

for $k=0,1,2, \cdots, n-1$.

Step 6. Calculate every $U_{k}, k=1,2, \cdots, n$ of an equation of degree two. ([ $\left.U_{0}, U_{1}, U_{2}, \cdots, U_{n}\right]$ are $F$-transform.)

Step 7. The approximate solution with IFT is

$$
u_{n}(t)=\sum_{k=0}^{n} U_{k} B_{k}(t)
$$

\subsection{Approximate solution of FBDEs}

In order to gain the approximate solution of the problem (1.2), we use of $u_{n}(t)$, hence

$$
D^{\alpha} u_{n}(t)=\lambda \exp \left(u_{n}(t)\right), \quad 1<\alpha \leq 2, \quad 0<t \leq T,
$$


and by putting $t=t_{k+1}$, we have

$$
\begin{aligned}
& D^{\alpha} u_{n}\left(t_{k+1}\right)=\lambda \exp \left(u_{n}\left(t_{k+1}\right)\right), \\
& \lambda>0, \quad 1<\alpha \leq 2, \quad k=0,1, \cdots, n-1 .
\end{aligned}
$$

Case 1. Considering Caputo's derivative: using Eq.(4.3), Eq.(4.11) convert to the following form

$\frac{1}{\tau^{\alpha} \Gamma(3-\alpha)} \sum_{j=0}^{k}\left(U_{j+1}-2 U_{j}+U_{j-1}\right)\left((k-j+1)^{2-\alpha}-(k-j)^{2-\alpha}\right)$

$$
=\lambda \exp \left(U_{k+1}\right), k=0,1,2, \cdots, n-1 \text {. }
$$

Case 2. Considering Caputo-Fabrizio derivative: using Eq.(4.5), Eq.(4.11) convert to the following form

$$
\begin{aligned}
& \frac{1}{\alpha \tau^{2}} \sum_{j=0}^{k}\left(U_{j+1}-2 U_{j}+U_{j-1}\right)\left(\exp \left(-\frac{(\alpha \tau)(k-j)}{1-\alpha}\right)\right. \\
& \left.-\exp \left(-\frac{(\alpha \tau)(k-j+1)}{1-\alpha}\right)\right)=\lambda \exp \left(U_{k+1}\right)
\end{aligned}
$$

in which $k=0,1,2, \cdots, n-1$.

Now, using the boundary condition, we can calculate $U_{1}, U_{2}, \cdots, U_{n}$ by recursive equation and then by IFT gain the approximate solution $u(t) \approx u_{n}(t)$ for Eq.(1.2).

An algorithm for approximation of FBDEs by this method stated in the next Algorithm.

Algorithm 2. An approximation algorithm for FBDEs

Step 1. Input $U_{0}=u(0)$ and $u_{t}(0)=u_{0}^{\prime}, n$ and $T$.

Step 2. Set $\tau \leftarrow \frac{T}{n}$ and $U_{-1} \leftarrow U_{0}-\tau u_{0}^{\prime}$.

Step 3. Locate $t_{k} \leftarrow k \tau, k=0,1,2, \cdots, n$.

Step 4. Choose sinusoidal BFs $B_{k}(t)$ for $k=0,1,2, \cdots, n$.

Step 5. a) With Caputo derivative, set recursive equation

$$
\begin{aligned}
& \frac{1}{\tau^{\alpha} \Gamma(3-\alpha)} \sum_{j=0}^{k}\left(U_{j+1}-2 U_{j}+U_{j-1}\right) \times \\
& \left((k-j+1)^{2-\alpha}-(k-j)^{2-\alpha}\right)=\lambda \exp \left(U_{k+1}\right) .
\end{aligned}
$$

b) With Caputo-Fabrizio derivative, set recursive equation

$$
\begin{aligned}
& \frac{1}{\alpha \tau^{2}} \sum_{j=0}^{k}\left(U_{j+1}-2 U_{j}+U_{j-1}\right)\left(\exp \left(-\frac{(\alpha \tau)(k-j)}{1-\alpha}\right)\right. \\
& \left.-\exp \left(-\frac{(\alpha \tau)(k-j+1)}{1-\alpha}\right)\right)=\lambda \exp \left(U_{k+1}\right),
\end{aligned}
$$

for $k=0,1,2, \cdots, n-1$.

Step 6. Calculate every $U_{k}, k=1,2, \cdots, n$ of an equation

of degree one. ( $\left[U_{0}, U_{1}, U_{2}, \cdots, U_{n}\right]$ are $F$-transform.)

Step 7. The approximate solution with IFT is

$$
u_{n}(t)=\sum_{k=0}^{n} U_{k} B_{k}(t)
$$

\section{Examples}

Now in this section, we present various examples for illustrate FTM for FRDEs and FBDEs. In all these examples, we used of mathematical software Mathematica.

Example 5.1. For the first example, we propose the FRDEs [43]:

$$
D_{t}^{\alpha} u(t)=1-u^{2}(t), \quad 0<t<1, \quad 0<\alpha \leq 1,
$$

with the precise solution $u(t)=\frac{\exp (2 t)-1}{\exp (2 t)+1}$ for $\alpha=1$ and the primary condition:

$$
u_{0}=u(0)=0
$$

Following the FTM, according to what was formulated and presented in section 4 for Eqs.(5.1)-(5.2), we can calculate $U_{1}, U_{2}, \ldots, U_{n}$ and then gain the approximate solution $u_{n}(t)$ of (5.1).

Table 1 shows comparison betwixt the exact and the approximation solution (5.1) with $F$-transform of test example 5.1 for different values of $\alpha$ and $t, n=500, \tau=$ 0.002, featuring Caputo and Caputo-Fabrizio derivative.

Comparison of exact and approximate solution can be seen for equations with different values of $\alpha, n=500$, $\tau=0.002$ and various values of $t$, in Figure 2 with Caputo derivative and in Figure 1 with Caputo-Fabrizo derivative.

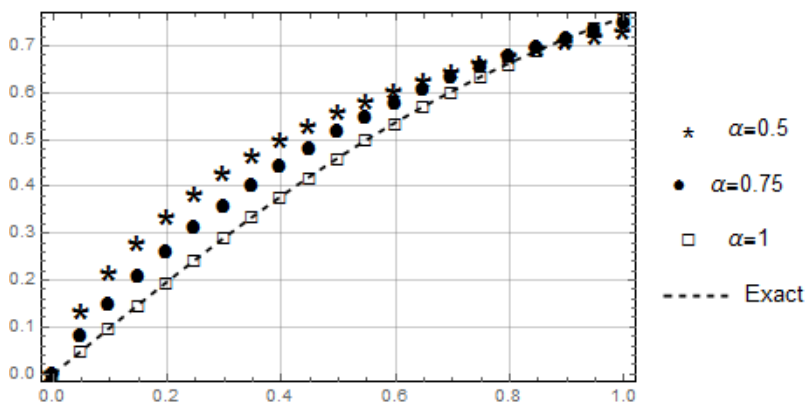

Fig. 1: Comparison betwixt the exact and the approximation solution with $F$-transform of test example 5.1 for $n=500, \tau=0.002$ and various values of $t$ and $\alpha$ with Caputo-Fabrizio derivative.

Table 2 represents the present method for $\alpha=1$ and the achieved results of homotopy perturbation method ( HPM), Adomian decomposition method (ADM) [30] and optimal homotopy asymptotic method (OHAM) [31]. 
Table 1: The exact and approximate result of test example 5.1 featuring various values of $\alpha$, with Caputo and Caputo-Fabrizio derivative.

\begin{tabular}{|c|c|c|c|c|c|c|c|c|}
\hline \multirow[b]{2}{*}{$t$} & \multicolumn{3}{|c|}{ Caputo } & \multirow[b]{2}{*}{ Exact } & \multicolumn{3}{|c|}{ Caputo-Fabrizio } & \multirow[b]{2}{*}{ Exact } \\
\hline & $\alpha=0.5$ & $\alpha=0.75$ & $\alpha=1.0$ & & $\alpha=0.5$ & $\alpha=0.75$ & $\alpha=1.0$ & \\
\hline 0.0 & 0.0 & 0.0 & 0.0 & 0.0 & 0.0 & 0.0 & 0.0 & 0.0 \\
\hline 0.2 & 0.334626 & 0.260941 & 0.197495 & 0.197375 & 0.334615 & 0.260932 & 0.197437 & 0.197375 \\
\hline 0.4 & 0.498466 & 0.442638 & 0.379972 & 0.379949 & 0.498459 & 0.442629 & 0.379895 & 0.379949 \\
\hline 0.6 & 0.604588 & 0.577781 & 0.536921 & 0.53705 & 0.604578 & 0.577780 & 0.536847 & 0.53705 \\
\hline 0.8 & 0.677429 & 0.67693 & 0.66383 & 0.664037 & 0.677418 & 0.67687 & 0.66377 & 0.664037 \\
\hline 1.0 & 0.729503 & 0.749104 & 0.761407 & 0.761594 & 0.729502 & 0.749103 & 0.76137 & 0.761594 \\
\hline
\end{tabular}

Table 2: Comparison of the numerical solutions of the equation in example 5.1 with $\alpha=1$.

\begin{tabular}{ccccccc}
\hline & & & & \multicolumn{3}{c}{ FTM } \\
\cline { 5 - 6 }$t$ & HPM & ADM & OHAM & Caputo & Caputo-Fabrizio & Exact \\
\hline 0.2 & 0.197375 & 0.197375 & 0.197402 & 0.197437 & 0.197437 & 0.197375 \\
0.4 & 0.379943 & 0.379948 & 0.380065 & 0.379972 & 0.379895 & 0.379949 \\
0.6 & 0.536857 & 0.537049 & 0.537148 & 0.536921 & 0.536847 & 0.53705 \\
0.8 & 0.661706 & 0.664037 & 0.664049 & 0.66383 & 0.66377 & 0.664037 \\
1.0 & 0.7460318 & 0.761622 & 0.761634 & 0.761407 & 0.76137 & 0.761594 \\
\hline
\end{tabular}

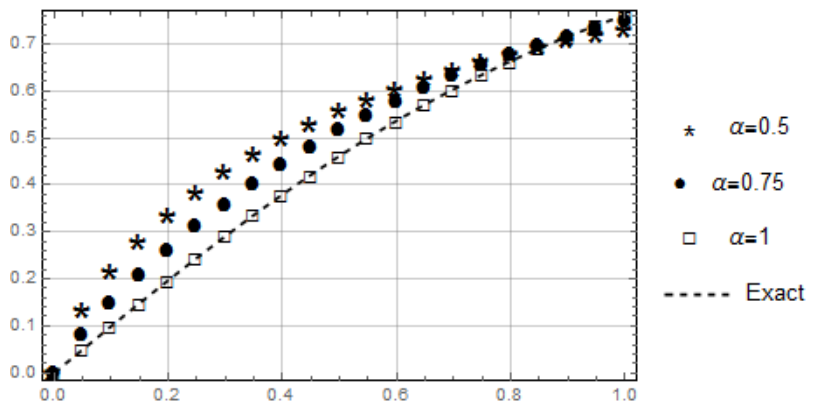

Fig. 2: Comparison betwixt the exact and the approximation solution with $F$-transform of test example 5.1 for value of $n=500$, $\tau=0.002$ and different values of $\alpha$ and $t$ with Caputo derivative.

Example 5.2. For the second example, we offer the FBDEs [32]:

$$
D_{t}^{\alpha} u(t)-2 \exp (u(t))=0, \quad 1<\alpha \leq 2
$$

including the primary condition

$$
u_{0}=u(0)=0, u_{0}^{\prime}=u_{t}(0)=0 .
$$

The unknown coefficient $U_{1}, U_{2}, \cdots, U_{n}$ with due attention to the FTM, according to section 4 for Eqs.(5.3)-(5.4) are calculated.

Comparison of exact and approximate solution can be seen in Table 3 for equations with $n=500, \tau=0.002$ and various values of $t$ and $\alpha$, featuring Caputo fractional derivative.

Figure 3 and Figure 4 shows comparison betwixt the exact and the approximation solution (5.1) with $F$-transform of test example 5.1 for various values of $\alpha, n=500, \tau=$

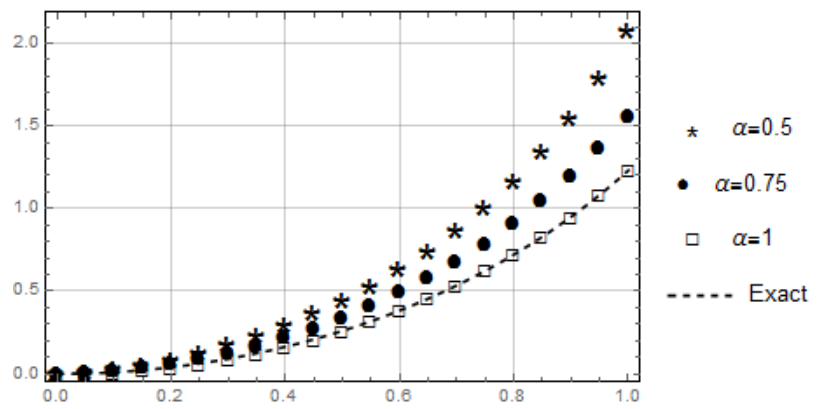

Fig. 3: Comparison betwixt the exact and the approximation solution with $F$-transform of test example 5.2 for different values of $\alpha$, $n=500, \tau=0.002$ and various values of $t$, with Caputo fractional derivative.

0.002, respectively, with Caputo and Caputo-Fabrizio fractional derivative.

Toward $\alpha=2$, the solution that we have gained is in accordance with the precise solution $u(t)=-2 \log (\cos (t))$.

Example 5.3. For the second example, we offer the FBDEs [32]:

$$
D_{t}^{\alpha} u(t)+2 \exp (u(t))=0, \quad 1<\alpha \leq 2
$$

including the primary condition

$$
u_{0}=u(0)=0, u_{0}^{\prime}=u_{t}(0)=0 .
$$

Toward $\alpha=2$, the solution that we have gained is in accordance with the precise solution $u(t)=$ $-2 . \log (0.848338 \cosh (1.17878(t-0.5)))$.

Table 4 represents the present method for $\alpha=2$ and the achieved results of Laplace transform method (LTM), 
Table 3: The exact and approximate result of test example 5.2 featuring various values of $\alpha$, with Caputo and Caputo-Fabrizio derivative.

\begin{tabular}{|c|c|c|c|c|c|c|c|c|}
\hline \multirow[b]{2}{*}{$t$} & \multicolumn{3}{|c|}{ Caputo } & \multirow[b]{2}{*}{ Exact } & \multicolumn{3}{|c|}{ Caputo-Fabrizio } & \multirow[b]{2}{*}{ Exact } \\
\hline & $\alpha=0.5$ & $\alpha=0.75$ & $\alpha=1.0$ & & $\alpha=0.5$ & $\alpha=0.75$ & $\alpha=1.0$ & \\
\hline 0.0 & 0.0 & 0.0 & 0.0 & 0.0 & 0.0 & 0.0 & 0.0 & 0.0 \\
\hline 0.2 & 0.0859606 & 0.059272 & 0.0404369 & 0.0402695 & 0.0859605 & 0.059271 & 0.0404368 & 0.0402695 \\
\hline 0.4 & 0.292598 & 0.219867 & 0.164802 & 0.164458 & 0.292597 & 0.219866 & 0.164802 & 0.164458 \\
\hline 0.6 & 0.630075 & 0.490616 & 0.384455 & 0.38393 & 0.630076 & 0.490615 & 0.384454 & 0.38393 \\
\hline 0.8 & 1.15995 & 0.907069 & 0.723658 & 0.722781 & 1.15995 & 0.907070 & 0.723656 & 0.722781 \\
\hline 1.0 & 1.54487 & 1.19373 & 1.23315 & 1.23125 & 1.54487 & 1.19373 & 1.23315 & 1.23125 \\
\hline
\end{tabular}

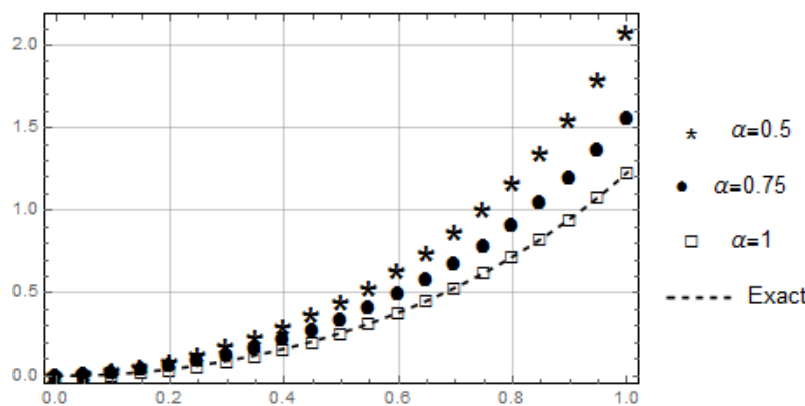

Fig. 4: Comparison betwixt the exact and the approximation solution with $F$-transform of test example 5.2 for value of $n=500$, $\tau=0.002$ and various values of $\alpha$ and $t$, with Caputo-Fabrizio derivative.

decomposition method (DM) and B-spline method (BSM) [58].

In this method, by increasing the amount $n$ and decreasing the amount $\tau$, a more accurate answer can be achieved. The time that the CPU is used in minutes for FRDEs with $\alpha=1$ and FBDEs with $\alpha=2$ featuring Caputo derivative and Caputo-Fabrizio derivative in difference, $\tau=0.002$, $n=50$ and $n=500$ is shown in Table 5. Baleanu et.al in [59] in a non-difference state compared the Caputo derivative and Caputo-Fabrizio derivative in terms of run-time in seconds.

\section{Conclusion}

We have successfully applied FTM to obtain approximate solution of the FRDEs and FBDEs. The result indicate that a few iteration of FTM will result in some useful solutions. Finally, it should be added that the suggested technique has the potentials to be practical in solving other similar nonlinear and linear problems in partial differential equations featuring fractional derivative.
Acknowledgement: We are very much indebted to Prof. Irina Perfilieva for constructive comments and helpful suggestions which led to improved presentation and quality of this paper.

\section{References}

[1] Magin R.L., Abdullah O., Baleanu D., Zhou X.J., Anomalous diffusion expressed through fractional order differential operators in the Bloch-Torrey equation, J. Magn. Reson., 2008, 190(2), 255-270.

[2] Scalas E., The application of continuous-time random walks in finance and economics, Phys. A: Stat. Mech. Appl., 2006, 362(2), 225-239.

[3] Deshpande A.S., Daftardar-Gejji V., Sukale Y.V., On Hopf bifurcation in fractional dynamical systems. Chaos, Solit. Fract., 2017, 98, 189-198.

[4] Neamaty A., Nategh M., Agheli B., Local non-integer order dynamic problems on time scales revisited, Int. J. Dyn. Contr., 2018, 6(2) , 486-498.

[5] Raja M.A.Z., Samar R., Alaidarous E.S., Shivanian E., Bioinspired computing platform for reliable solution of Bratu-type equations arising in the modeling of electrically conducting solids, Appl. Math. Model., 2016, 40(11), 5964-5977.

[6] Coronel-Escamilla A., Gómez-Aguilar J. F., Torres L., EscobarJiménez R.F., Valtierra-Rodríguez M., Synchronization of chaotic systems involving fractional operators of Liouville-Caputo type with variable-order, Phys. A: Stat. Mech. Appl., 2017, 487, 1-21.

[7] Guner O., Bekir A., The Exp-function method for solving nonlinear space-time fractional differential equations in mathematical physics, J. Assoc. Arab Univ. Basic Appl. Sci., 2017, 24, 277-282.

[8] Gómez-Aguilar J.F., Atangana A., New insight in fractional differentiation: power, exponential decay and Mittag- Leffler laws and applications, Europ. Phys. J. Plus, 2017, 132(1), 13.

[9] Coronel-Escamilla A., Gómez-Aguilar J.F., Torres L., EscobarJiménez R.F., A numerical solution for a variable-order reaction-diffusion model by using fractional derivatives with non-local and non-singular kernel, Phys. A: Stat. Mech. Appl., 2018, 491, 406-424.

[10] Atangana A., Gómez-Aguilar J.F., A new derivative with normal distribution kernel: Theory, methods and applications. Phys. A: Stat. Mech., Appl., 2017, 476, 1-14. 
Table 4: Comparison of the numerical solutions of the equation in example 5.3 with $\alpha=2$.

\begin{tabular}{ccccccc}
\hline & & & & \multicolumn{3}{c}{ FTM } \\
\cline { 5 - 6 }$t$ & LTM & DM & BSM & Caputo & Caputo-Fabrizio & Exact \\
\hline 0.5 & 0.319353 & 0.335937 & 0.328896 & 0.32758 & 0.327591 & 0.328952 \\
0.6 & 0.304160 & 0.318336 & 0.315036 & 0.313499 & 0.313483 & 0.315089 \\
0.7 & 0.261946 & 0.267991 & 0.273834 & 0.272173 & 0.272087 & 0.273879 \\
0.8 & 0.194041 & 0.191744 & 0.206386 & 0.204666 & 0.204517 & 0.206419 \\
0.9 & 0.103537 & 0.099193 & 0.114393 & 0.11262 & 0.112484 & 0.114411 \\
\hline
\end{tabular}

Table 5: Duration used in minutes.

\begin{tabular}{cccccc}
\hline & \multicolumn{2}{c}{ FRDEs } & & \multicolumn{2}{c}{ FBDEs } \\
\cline { 2 - 3 } \cline { 5 - 6 } & Caputo & Caputo-Fabrizio & & Caputo & Caputo-Fabrizio \\
\hline$n=50$ & 0.0369792 & 0.0403646 & & 0.0914063 & 0.09375 \\
$n=500$ & 5.61693 & 5.62161 & & 7.07031 & 7.65938 \\
\hline
\end{tabular}

[11] Morales-Delgado V.F., Taneco-Hernández M.A., Gómez- Aguilar J.F., On the solutions of fractional order of evolution equations, Europ. Phys. J. Plus, 2017, 132(1), 47.

[12] Neamaty A., Nategh M., Agheli B., Time-Space Fractional Burger's Equation on Time Scales, J. Comput. Nonlin Dyn., 2017, 12(3), 031022.

[13] Coronel-Escamilla A., Gómez-Aguilar J. F., Baleanu D., Córdova-Fraga T., Escobar-Jiménez R.F., Olivares-Peregrino V.H., Qurashi M.M.A., Bateman-Feshbach Tikochinsky and Caldirola-Kanai Oscillators with New Fractional Differentiation, Entropy, 2017, 19(2), 55.

[14] Ming C., Liu F., Zheng L., Turner I., Anh V., Analytical solutions of multi-term time fractional differential equations and application to unsteady flows of generalized viscoelastic fluid, Comp. Math. Appl., 2016, 72(9), 2084-2097.

[15] Baleanu D., Luo A.C., Discontinuity and Complexity in Nonlinear Physical Systems, J.T. Machado (Ed.), 2014, Springer.

[16] Kilbas A.A., Srivastava H.M., Trujillo J.J., Theory and application of fractional differential equations, 2006, Elsevier B.V, Netherlands.

[17] Caputo M., Fabrizio, M., A new definition of fractional derivative without singular kernel, Progr. Fract. Differ. Appl, 2015, 1(2), 1-13.

[18] Reid W.T., Riccati Differential Equations (Mathematics in Science and Engineering), 1972, 86, Academic Press, New York.

[19] Ntogramatzidis L., Ferrante, A., On the solution of the Riccati differential equation arising from the LQ optimal control problem, Syst. Contr. Lett., 2010, 59(2), 114-121.

[20] Anderson B.D., Moore J.B., Optimal filtering, Englewood Cliffs, 1979, 21, 22-95.

[21] Gerber M., Hasselblatt B., Keesing D., The Riccati equation: pinching of forcing and solutions, Exp. Math., 2003, 12(2), 129-134.

[22] Einicke G.A., White L.B., Bitmead R.R., The use of fake algebraic Riccati equations for co-channel demodulation, IEEE transactions on signal processing, 2003, 51(9), 2288-2293.

[23] Boyle P.P., Tian W., Guan F., The Riccati equation in mathematical finance, J. Symbol. Comput., 2002, 33(3), 343-355.

[24] Lasiecka I., Triggiani R., Differential and algebraic Riccati equations with application to boundary/point control problems: continuous theory and approximation theory, Lecture notes in control and Information Sciences, 1991, 164, 1-160.

[25] Anderson B.D., Moore J.B., Optimal control: linear quadratic methods, 2007, Prentice-Hall, New Jersey.

[26] Odibat Z., A Riccati Equation Approach and Travelling Wave Solutions for Nonlinear Evolution Equations, Int. J. Appl. Comput. Math., 2017, 3(1), 1-13.

[27] Kravchenko V.V., Applied pseudoanalytic function Theory, 2009, Springer Science \& Business Media.

[28] Jacobsen J., Schmitt K., The Liouville-Bratu-Gelfand problem for radial operators, J. Diff. Equat., 2002, 184(1), 283-298.

[29] Frank-Kamenetskii D.A., Diffusion and heat exchange in chemical kinetics, 2015, Princeton University Press.

[30] Abbasbandy S., Homotopy perturbation method for quadratic Riccati differential equation and comparison with Adomian's decomposition method, Appl. Math. Comput., 2006, 172(1), 485-490.

[31] Riccati H.P., Mabood F., Izani A., Ismai M., Hashim I., Application of optimal homotopy asymptotic method for the approximate solution of Riccati equation, Sains Malaysiana, 2013, 42(6), 863-867.

[32] Ragb 0., Seddek L.F., Matbuly M.S., Iterative differential quadrature solutions for Bratu problem, Comp. Math. Appl., 2017, 74(2), 249-257.

[33] Jator S.N., Manathunga V., Block Nyström type integrator for Bratu's equation, J. Comput. Appl. Math., 2018, 327, 341-349.

[34] Khastan A., Perfilieva I., Alijani Z., A new fuzzy approximation method to Cauchy problems by fuzzy transform, Fuzzy Sets Syst., 2016, 288, 75-95.

[35] Khastan A., Alijani Z., Perfilieva I., Fuzzy transform to approximate solution of two-point boundary value problems, Math. Meth. Appl. Sci., 2016, 40(17), 6147-6154.

[36] Tomasiello S., An alternative use of fuzzy transform with application to a class of delay differential equations, Int. J. Comp. Math., 2016, 94(9), 1719-1726.

[37] Chen W., Shen Y., Approximate solution for a class of secondorder ordinary differential equations by the fuzzy transform, J. Intel. Fuzzy Syst., 2014, 27(1), 73-82.

[38] Biazar J., Eslami M., Differential transform method for quadratic Riccati differential equation, Int. J. Nonlin. Sci., 2010, 9(4), 444-447. 
[39] Abbasbandy S., Homotopy perturbation method for quadratic Riccati differential equation and comparison with Adomian's decomposition method, Appl. Math. Comput., 2006, 172(1), 485-490.

[40] Geng F., A modified variational iteration method for solving Riccati differential equations, Comp. Math. Appl., 2010, 60(7), 1868-1872.

[41] Tan Y., Abbasbandy S., Homotopy analysis method for quadratic Riccati differential equation, Comm. Nonlin. Sci. Numer. Simul., 2008, 13(3), 539-546.

[42] Bota C., Căruntu B., Analytical approximate solutions for quadratic Riccati differential equation of fractional order using the Polynomial Least Squares Method, Chaos, Solit. Fractals, 2017, 102, 339-345.

[43] Neamaty A., Agheli B., Darzi R., The shifted Jacobi polynomial integral operational matrix for solving Riccati differential equation of fractional order, Appl. Appl. Math., 2015, 10(2), 878-892.

[44] Maleknejad K., Torkzadeh L., Hybrid Functions Approach for the Fractional Riccati Differential Equation, Filomat, 2016, 30(9), 2453-2463.

[45] Aminikhah H., Sheikhani A.H.R., Rezazadeh H., Approximate analytical solutions of distributed order fractional Riccati differential equation, Ain Shams Eng. J., 2016, 9(4), 581-588.

[46] Feng X., He Y., Meng J., Application of homotopy perturbation method to the Bratu-type equations, Topol. Meth. Nonlin. An., 2008, 31(2), 243-252.

[47] Darwish M.A., Kashkari B.S., Numerical solutions of second order initial value problems of Bratu-type via optimal homotopy asymptotic method, Amer. J. Comput. Math., 2014, 4(02), 47.

[48] Das N., Singh R., Wazwaz A.M., Kumar J., An algorithm based on the variational iteration technique for the Bratu-type and the Lane-Emden problems, J. Math. Chem., 2016, 54(2), 527551.

[49] Morales-Delgado V.F., Gómez-Aguilar J.F., Yépez-Martínez H., Baleanu D., Escobar-Jimenez R.F., Olivares-Peregrino V.H., Laplace homotopy analysis method for solving linear partial differential equations using a fractional derivative with and without kernel singular, Adv. Diff. Equat., 2016, 2016(1), 164.
[50] Gómez-Aguilar J.F., Yépez-Martínez H., Torres-Jiménez, J., Córdova-Fraga T., Escobar-Jiménez R.F., Olivares- Peregrino V.H., Homotopy perturbation transform method for nonlinear differential equations involving to fractional operator with exponential kernel, Adv. Diff. Equat., 2017, 2017(1), 68.

[51] Atangana A., Gómez-Aguilar J.F., Numerical approximation of Riemann-Liouville definition of fractional derivative: From Riemann-Liouville to Atangana-Baleanu, Num. Meth. Part. Diff. Equat., 2018, 34(5), 1502-1523.

[52] Yépez-Martínez H., Gómez-Aguilar J.F., Sosa I.O., Reyes J.M., Torres-Jiménez J., The Feng's first integral method applied to the nonlinear mKdV space-time fractional partial differential equation, Rev. Mex. Física, 2016, 62(4), 310-316.

[53] Yépez-Martínez H., Gómez-Aguilar J.F., Atangana A., First integral method for non-linear differential equations with conformable derivative, Math. Model. Nat. Phenom., 2018, 13(1), 14.

[54] Yépez-Martínez H., Gómez-Aguilar J.F., Numerical and analytical solutions of nonlinear differential equations involving fractional operators with power and Mittag-Leffler kernel, Math. Model. Nat. Phenom., 2018, 13(1), 13.

[55] Perfilieva I., Fuzzy transforms: Theory and applications. Fuzzy sets and systems, 2006, 157(8), 993-1023.

[56] Li C., Zhao Z., Chen Y., Numerical approximation of nonlinear fractional differential equations with subdiffusion and superdiffusion, Comp. Math. Appl., 2011, 62(3), 855-875.

[57] Atangana A., Alqahtani R.T., Numerical approximation of the space-time Caputo-Fabrizio fractional derivative and application to groundwater pollution equation, Adv. Diff. Equat., 2016, 2016(1), 156.

[58] Caglar H., Caglar N., Özer M., Valarıstos A., Anagnostopoulos A.N., B-spline method for solving Bratu's problem, Int. J. Comp. Math., 2010, 87(8), 1885-1891.

[59] Baleanu D., Agheli B., Al Qurashi M.M., Fractional advection differential equation within Caputo and Caputo- Fabrizio derivatives, Adv. Mech. Eng., 2016, 8(12), 1687814016683305. 